\title{
S100B regulates inflammatory response during osteoarthritis via fibroblast growth factor receptor 1 signaling
}

\author{
LIFAN ZHU $^{1 *}$, ZHEN WENG $^{2 *}$, PENGCHENG SHEN $^{1 *}$, JIANXIN ZHOU $^{1}$, JINCAI ZENG $^{1}$, \\ FENGBIAO WENG ${ }^{1}$, XIAOJIAN ZHANG ${ }^{3}$ and HUILIN YANG ${ }^{4}$ \\ ${ }^{1}$ Department of Orthopaedics, The First People's Hospital of Wujiang, Suzhou, Jiangsu 215200; \\ ${ }^{2}$ Cyrus Tang Hematology Center, Soochow University, Suzhou, Jiangsu 215006; ${ }^{3}$ Department of Surgery, \\ The First People's Hospital of Wujiang, Suzhou, Jiangsu 215200; ${ }^{4}$ Department of Orthopaedics, \\ The First Affiliated Hospital of Soochow University, Suzhou, Jiangsu 215006, P.R. China
}

Received December 27, 2017; Accepted September 5, 2018

DOI: $10.3892 / \mathrm{mmr} .2018 .9523$

\begin{abstract}
The present study aimed to investigate the role of S100B in the inflammation process during osteoarthritis (OA). OA cartilage samples were collected for S100B expression analysis. S100B expression levels were significantly increased in patients with OA compared with the Controls $(1.28 \pm 0.66$ vs. $0.42 \pm 0.31 ; \mathrm{P}=0.01)$ and were determined to be correlated with TNF- $\alpha(r=0.42 ; \mathrm{P}=0.04)$ and IL- $1 \beta(\mathrm{r}=0.73$; $\mathrm{P}=0.001)$ expression levels. Orthopedic casting tape was used to immobilize the right knee at $180^{\circ}$ extension of adult female New Zealand white rabbits for 4 weeks to establish an OA model. Cartilage specimens from the medial femoral condyle of these rabbits were used for histological confirmation and immunohistochemical analyses, whereas synovial fluid was used in ELISA assays for tumor necrosis factor (TNF)- $\alpha$ and interleukin (IL)-1 $1 \beta$ expression levels. Human synovial fibroblasts from the knee synovial tissues of normal patients with traumatic injury were transfected with S100B overexpression and knockdown plasmids and subjected to lipopolysaccharide (LPS) stimulation; subsequently, TNF- $\alpha$ and IL-1 $\beta$ expression levels in conditioned medium were determined by ELISA; S100B overexpression and knockdown significantly increased and decreased the TNF- $\alpha$ and IL-1 $\beta$ expression levels, respectively. Increased TNF- $\alpha(573.3 \pm 15.4$ vs. $102.6 \pm 8.7 \mathrm{pg})$ and IL-1 $\beta(378.6 \pm 7.2$ vs. $170.1 \pm 5.8 \mathrm{pg})$ expression levels were detected in OA model rabbits compared with the Control rabbits. Additionally, S100B, fibroblast growth factor (FGF)-1 and FGF receptor (FGFR)-1 mRNA and protein expression
\end{abstract}

Correspondence to: Dr Huilin Yang, Department of Orthopaedics, The First Affiliated Hospital of Soochow University, 188 Shizi Street, Suzhou, Jiangsu 215006, P.R. China

E-mail: szspineyhl@163.com

${ }^{*}$ Contributed equally

Key words: S100B, osteoarthritis, fibroblast growth factor receptor 1, inflammation levels were increased in OA model rabbits compared with the Control group. FGFR1 knockdown significantly decreased TNF- $\alpha$ and IL-1 $\beta$ expression levels in LPS-stimulated S100B-overexpressing human synovial fibroblasts. S100B is involved in FGFR1 signaling-mediated inflammatory response during OA, which may be considered as a potential therapeutic target.

\section{Introduction}

Osteoarthritis (OA) is one of the most common types of joint disease, particularly in the elderly, with $\sim 3$ million newly diagnosed cases each year $(1,2)$. Owing to limited information about the pathophysiological process of OA, joint replacement remains the main treatment for patients with advanced OA (3), and only limited effective disease-modifying OA drugs have been used for treatment (4). Further understanding of the mechanism underlying OA may provide insights for the development of novel treatments.

Previous studies have demonstrated that inflammation contributes to the pathogenesis and the progression of OA $(5,6)$, and is a major factor associated with cartilage loss and disease symptoms, including joint pain, swelling and stiffness, as well as synovitis indications, such as joint tenderness and abscesses (7). Infiltration of mononuclear cells into the synovial membrane and production of proinflammatory mediators, such as interleukin $1 \beta$ (IL-1 $\beta$ ), tumor necrosis factor- $\alpha$ (TNF- $\alpha$ ) and chemokines, may initiate and exacerbate OA (7). Exploration of the mechanisms and identification of the key mediators involved in the inflammatory process, particularly to those associated with IL- $1 \beta$ and TNF- $\alpha$, will aid in prevention and management of OA.

S100B is a $21 \mathrm{kDa}$ EF hand-type cytosolic calcium-binding protein that has been demonstrated to serve a stimulatory role for inflammatory responses in multiple cells, such as macrophages, lymphocytes, endothelial cells, vascular smooth muscle cells and cardiomyocytes $(8,9)$. According to previous studies $(10,11)$, S100B has been revealed to be expressed in human articular cartilage, and the expression of S100B is upregulated in diseased tissue. In addition, a previous study suggested that extracellular S100B is a pro-catabolic and 
pro-inflammatory factor that promotes cartilage degradation (11). However, the exact role of S100B in OA as well its association with important inflammatory factors, such as IL-1 $\beta$ and TNF- $\alpha$, have not been investigated. Therefore, the aim of the present study was to explore the role of S100B in the inflammation process during OA. Furthermore, lipopolysaccharides (LPS), an important proinflammatory product of the microbiome, has a role in the pathogenesis of OA, and both systemic and local LPS burden is considered to be associated with knee OA (12). Therefore, LPS was as the stimulator in the cell culture system used in the present study (12).

\section{Materials and methods}

Ethics approval of the study protocol. All research involving human participants was approved by the Institutional Review Board of Soochow University (Suzhou, China). Written informed consent was provided by all participants. The animal study protocol was approved by the Institutional Animal Care and Use Committee of Soochow University and was conducted following the international guidelines for animal experimentation (13).

OA cartilage samples. OA cartilage samples $(n=23$; age $74.0 \pm 3.9$ years old; sex, 21 females and 2 males) were collected between January and December 2016 during total knee arthroplasty in the Department of Orthopaedics at The First Affiliated Hospital of Soochow University. Inclusion criteria were as follows: Patients were diagnosed with OA in accordance with the definition and classification provided by the Diagnostic and Therapeutic Criteria Committee of the American Rheumatism Association (14); patients with rheumatoid arthritis and other autoimmune diseases, as well as chondrodysplasias and posttraumatic OA were excluded from the study. For RNA extraction, cartilage samples (2-3 g) were collected in $1.5 \mathrm{ml}$ centrifugation tube, grinded with liquid nitrogen, mixed with $1 \mathrm{ml} \mathrm{TRIzol}{ }^{\circledR}$ (Invitrogen; Thermo Fisher Scientific, Inc., Waltham, MA, USA) and stored in $-80^{\circ} \mathrm{C}$ in the absence of extraction prior to subsequent use. For immunohistochemical analyses, cartilage samples were fixed with $10 \%$ neutral formalin prior to further processing. A total of 20 samples of articular cartilage were obtained from patients (age, $71.7 \pm 3.6$ years old; sex, 18 females and 2 males) with tibial plateau fracture and without $\mathrm{OA}$ were also collected as control between January and December 2016 at the same hospital. Written informed consent was obtained from all OA and control subjects. The patient demographic data, including age, sex, disease duration and Kellgren-Lawrence grading are listed in Table I.

Rabbit OA model establishment. Female adult New Zealand white rabbits $(\mathrm{n}=9,6$ rabbits were used to establish the OA model and 3 rabbits were used as control; age, 4 months; weight, 3.2-3.8 kg) were purchased from Shanghai SLAC Laboratory Animal Co., Ltd (Shanghai, China), housed in an air-conditioned room at $22^{\circ} \mathrm{C}$ with a $12 / 12 \mathrm{~h} \mathrm{light/dark}$ cycle and $40-60 \%$ humidity. Rabbits were permitted free access to standard laboratory food and water, and underwent right knee immobilization at $180^{\circ}$ of extension using orthopedic casting tape (Nanjing Shuangwei Biotech Co., Ltd., Jiangsu, China).
Normal weight bearing was carried out in all immobilized rabbits. Following 4 weeks immobilization, the tape was removed and cartilage samples were collected for experimentation. Successful establishment of the OA model was confirmed by visual observation of roughness of articular cartilage at 4 weeks in 5 rabbits and the efficacy was $83.3 \%$ (5 out of 6 rabbits were successfully established into to OA model; roughness of articular cartilage was not observed in the remaining rabbit, which suggested that OA was not successfully established). In addition, 3 rabbits without right knee immobilization were employed as normal Control. Therefore, 5 and 3 rabbits were used as the OA model and the control group, respectively.

Sample collection and histological assessment. The intermediate region of the medial femoral condyles of the OA and control osteochondral samples obtained from humans and rabbits were fixed with $10 \%$ neutral formalin at $22^{\circ} \mathrm{C}$ for $24 \mathrm{~h}$, followed by $5 \%$ nitric acid decalcification for 4 days, dehydration in a graded ethanol series at $22^{\circ} \mathrm{C}(70,80,90$ and $100 \%$; each for $10 \mathrm{~min}$ ), and then xylene clearance was performed using the following steps: Incubation at $60^{\circ} \mathrm{C}$ for $10 \mathrm{~min}$ and then two incubations at $22^{\circ} \mathrm{C}$ for $10 \mathrm{~min}$. Following this, sections were embedded in paraffin at $22^{\circ} \mathrm{C}$, and then cut into sections $(5 \mu \mathrm{m})$. Sections were stained with hematoxylin and eosin (H\&E) for general examination and Safranin-O/Fast-Green (SO) for glycosaminoglycan distribution was performed on the sections. Staining results in four visual fields were evaluated separately using a light microscope (Nikon Corporation, Tokyo, Japan). OA severity was evaluated by the Mankin and Osteoarthritis Research Society International scoring system (15).

Synovial fluid was obtained from the knee joint of OA and Control rabbits by injecting $0.5 \mathrm{ml}$ saline solution followed by aspiration, which was performed three times. Synovial fluid $(\sim 0.8-1 \mathrm{ml})$ was extracted and samples were centrifuged at $2,200 \mathrm{x} g$ for $10 \mathrm{~min}$. The supernatant was collected and stored at $-70^{\circ} \mathrm{C}$ until further use.

Immunohistochemical evaluation. Human OA and control sections were treated for $30 \mathrm{~min}$ with proteinase $\mathrm{K}$ (Kangchen BioTech Co., Ltd., Shanghai, China), 10 min with 3\% hydrogen peroxide/methanol and $30 \mathrm{~min}$ with $10 \%$ goat serum (Vector Laboratories, Inc., Burlingame, CA, USA). Sections were incubated with mouse primary antibodies against TNF- $\alpha$ (1:100; cat. no. sc-52746; Santa Cruz Biotechnology Inc., Dallas, TX, USA); IL-1 $\beta$ (1:100; cat. no. sc-7884; Santa Cruz Biotechnology Inc.), type II collagen (1:100; cat. no. NB600-844; Novus Biologicals, LLC, Littleton, CO, USA) and S100B (1:100; cat. no. HPA015768; Sigma-Aldrich; Merck KGaA, Darmstadt, Germany) for $90 \mathrm{~min}$. Subsequently, the sections were incubated with a secondary goat anti-mouse antibody (1:200; cat. no. KGAA37; Nanjing KeyGen Biotech. Co., Ltd., Nanjing, China) for $30 \mathrm{~min}$ at room temperature, followed by $5 \mathrm{~min}$ 3,3'-diaminobenzidine tetrahydrochloride solution staining, 8 min hematoxylin counterstaining and mounted using neutral balsam. A light microscope was used for evaluation and four distinct visual fields were observed. Digital images were subsequently analyzed using ImageJ software version $1.42 \mathrm{q}$ (National Institute of Health, Bethesda, MD, USA), according to previous studies $(16,17)$, and the results are expressed as relative intensity of staining. 
Table I. Clinicopathological characteristics of the patients with OA used in the present study.

\begin{tabular}{lccr}
\hline Characteristic & OA & Control & P-value \\
\hline Patients (n) & 23 & 20 & \\
K/L Grading & & & \\
Grade 2+3 & 4 & & \\
Grade 4 & 19 & & \\
Age (years) & $74.0 \pm 3.9$ & $71.7 \pm 3.6$ & 0.24 \\
Sex (F/M) & $21 / 2$ & $18 / 2$ & 0.70 \\
Disease duration (months) & $140.6 \pm 80.8$ & & \\
\hline
\end{tabular}

F, female; K/L, Kellgren-Lawrence; M, male; OA, osteoarthritis.

Human synovial fibroblast isolation and culture. Knee synovial tissues were obtained from normal patients with traumatic injury (as aforementioned) and human synovial fibroblasts were prepared using outgrowth technology, as described in a previous study (16). Briefly, synovial tissue samples were washed twice with PBS, minced into $1 \mathrm{~mm}$ pieces and placed in a $35 \mathrm{~mm}$ tissue culture dish and incubated in Ham's F12 medium supplemented with $10 \%$ FBS and penicillin/streptomycin (100 U/ml; all purchased from Gibco; Thermo Fisher Scientific, Inc., Waltham, MA, USA). The medium was changed every 3-4 days. Following confluency (80-90\%), cells were detached with $0.05 \%$ trypsin/EDTA solution and subcultured at a ratio of 1:3 (one plate was split into three plates). Human synovial fibroblasts were used between passage four and eight.

Lentiviral short interfering (si)RNA and overexpression vectors. Recombinant lentiviral particles overexpressing S100B or siRNA against S100B or fibroblast growth factor receptor 1 (FGFR1), and the controls (empty vector or Scrambled siRNA; sequences are provided in Table II). pLVX-IRES-ZsGreen1 was used as the overexpression vector, whereas pLVX-shRNA2 was used as the siRNA vector (both obtained from GenePharma Inc., Shanghai, China). Cells were grown to $40 \%$ confluency and transduced with complete medium containing lentiviral particles overexpressing S100B or siRNA against S100B or FGFR1 at concentrations of $1 \times 10^{8}$ transducing units $/ \mathrm{ml}$ [multiplicity of infection (MOI) of 20] (18) at $37^{\circ} \mathrm{C}$ for $48 \mathrm{~h}$. Polybrene (cat. no. H9268; Sigma-Aldrich; Merck KGaA) at a concentration of $8 \mu \mathrm{g} / \mathrm{ml}$ was added simultaneously to increase infection efficiency. No adverse effects were observed on the cell viability by siRNA or Polybrene (data not shown). The siRNAs had no off-target effects, and did not affect cell adherence, shape and viability at the MOI of 20, according to manufacturer's protocol and treatment duration.

Cell treatment and LPS simulation. To observe the effects of S100B on inflammatory cytokines, human synovial fibroblasts were infected with S100B overexpression, S100B siRNA, Scrambled siRNA or empty vector lentivirus as aforementioned, and subjected to LPS stimulation at $20 \mu \mathrm{g} / \mathrm{l}$ for $48 \mathrm{~h}$, followed by cell lysing using 200-500 $\mu \mathrm{l}$ radioimmunoprecipitation assay
Table II. Primer sequences used for reverse transcriptionquantitative polymerase chain reaction and siRNA sequences.

\begin{tabular}{ll}
\hline Gene & \multicolumn{1}{c}{ Primer sequence $\left(5^{\prime}-3 '\right)$} \\
\hline S100B & F: CTGGAGAAGGCCATGGTTGC \\
& R: CTCCAGGAAGTGAGAGAGCT \\
TNF- $\alpha$ & F: GGAGAAGGGTGACCGACTCA \\
& R: CTGCCCAGACTCGGCAA \\
IL-1 $\beta$ & F: AAGCTGATGGCCCTAAACAG \\
& R: AGGTGCATCGTGCACATAAG \\
FGF1 & F: ACAAGGGACAGGAGCGAC \\
& R: TCCAGCCTTTCCAGGAACA \\
FGFR1 & F: TTCCTCATCTCCTGCATGGT \\
& R: GTGGTGCTGAGTGTGCAAAT \\
GAPDH & F: CAAAGCCAGAGTCCTTCAGA \\
& R: GATGGTCTTGGTCCTTAGCC
\end{tabular}

Gene

siRNA sequence (5'-3')

S100B

FGF

Scramble

GGAATTCATGGCCTTTGTT

CCACAGAATTGGAGGCTACAA CCGTATCGTAAGCAGTACTTT

IL-1 $\beta$, interleukin 1 $\beta$; FGF1, fibroblast growth factor 1; FGFR1, fibroblast growth factor receptor 1 ; TNF- $\alpha$, tumor necrosis factor $\alpha$.

(RIPA) buffer (Beyotime Institute of Biotechnology, Haimen, China) at $4{ }^{\circ} \mathrm{C}$ and subsequently subjected to centrifugation at $14,000 \mathrm{x} \mathrm{g}$ for $15 \mathrm{~min}$ at $4^{\circ} \mathrm{C}$ to separate the total protein. In addition, condition medium was collected following further centrifugation at $1,500 \mathrm{xg}$ for $15 \mathrm{~min}$ at $4^{\circ} \mathrm{C}$. To observed the effects FGFR1 on inflammatory cytokines, human synovial fibroblasts were infected with S100B overexpression or empty vector lentivirus alone or co-transfected with FGFR1 siRNA or scramble siRNA lentivirus, stimulated with $20 \mu \mathrm{g} / 1 \mathrm{LPS}$ for $48 \mathrm{~h}$ before cell lysis and condition medium collection, as aforementioned.

ELISA. TNF- $\alpha$ and IL-1 $\beta$ expression levels from OA model and Control rabbit synovial fluid or condition medium of human synovial fibroblasts following cell treatment and LPS simulation were detected using the following ELISA kits purchased from R\&D Systems, Inc. (Minneapolis, MN, USA): Rabbit TNF- $\alpha$ kit (cat. no. DY5670), human TNF- $\alpha$ kit (cat. no. DY210-05), rabbit IL-1 $\beta$ kit (cat. no. DY7464) and human IL-1 $\beta$ kit (cat. no. DY201-05). Following this, absorbance values were measured at $450 \mathrm{~nm}$ to determine the concentrations, according to the manufacturer's protocol.

Reverse transcription-quantitative polymerase chain reaction (RT-qPCR) assay. Total RNA was extracted from human synovial fibroblasts $\left(1 \times 10^{6}\right)$ that had previously undergone different treatments, or osteochondral samples from control and OA rabbits, using TRIzol ${ }^{\circledR}$ reagent (Invitrogen; Thermo Fisher Scientific, Inc.). One Step SYBR PrimeScript RT-PCR 
kit (Takara Biotechnology Co., Ltd., Dalian, China) and an iQ5 Real-Time PCR Detection System (Bio-Rad Laboratories, Inc., Hercules, CA, USA) were used for PCR. The temperature protocol used for RT was $42^{\circ} \mathrm{C}$ for 5 min followed by $95^{\circ} \mathrm{C}$ for $10 \mathrm{sec}$. The following thermocyling conditions were used for qPCR: 40 cycles at $95^{\circ} \mathrm{C}$ for $5 \mathrm{sec}$ followed by $60^{\circ} \mathrm{C}$ for $30 \mathrm{sec}$; followed by a final termination step at $4^{\circ} \mathrm{C}$ for $10 \mathrm{~min}$. Relative gene expression was determined by the $2^{-\Delta \Delta \mathrm{Cq}}$ method (19) and expression level of the GAPDH gene from the same samples was used as an internal control. Specific oligonucleotide primers for S100B, TNF- $\alpha$, IL-1 $\beta$, FGFR1 and GAPDH are listed in Table II.

Western blot analysis. Human synovial fibroblasts or cells ( $1 \times 10^{7}$ cells) in cartilage tissue (2-3 g) from OA model or Control rabbits were lysed in radioimmunoprecipitation assay buffer (RIPA; Beyotime Institute of Biotechnology, Nantong, China), and extracted by supernatant collection following high speed centrifugation at $14,000 \mathrm{x}$ g for $15 \mathrm{~min}$ at $4^{\circ} \mathrm{C}$, followed by bicinchoninic acid assay for protein quantification. Cellular proteins $(20 \mu \mathrm{g})$ were separated by $10 \%$ SDS-PAGE gel and transferred onto polyvinylidene difluoride membranes. Subsequently, the membranes were blocked with non-fat milk and incubated with primary monoclonal antibodies at $4^{\circ} \mathrm{C}$ overnight against S100B (cat. no. 9550), FGF1 (cat. no. 3139) and FGFR1 (cat. no. 9740; all from Cell Signaling Technology, Inc., Danvers, MA, USA); and anti- $\beta$-actin antibody (Santa Cruz Biotechnology Inc.) was used as the loading control. Protein bands were incubated with horseradish peroxidase-conjugated secondary antibodies (cat. no. 7074; 1:2,000; Cell Signaling Technology, Inc.) at room temperature for $1 \mathrm{~h}$ and developed with SuperSignal Ultra Chemiluminescent Substrate (Pierce; Thermo Fisher Scientific, Inc.) and exposed on X-ray films (Kodak, Rochester, NY, USA). Image J software version 1.42 q (National Institutes of Health) was used for densitometric analysis, and protein expression levels were normalized against $\beta$-actin.

Statistical analysis. All experiments were performed at least three times. SPSS v18 (SPSS, Inc., Chicago, IL, USA) was used for statistical analysis. Data are presented as the mean \pm standard deviation. Between-group comparisons were performed by using Student's t-test or one-way analysis of variance followed by Tukey's post hoc test. Correlation analysis was performed with Pearson's correlation. $\mathrm{P}<0.05$ was considered to indicate a statistically significant difference.

\section{Results}

S100B, TNF- $\alpha$ and IL-expression in human OA tissues. Expression analysis of S100B, TNF- $\alpha$ and IL- $1 \beta$ was performed in human OA tissues (Fig. 1A; Table III). The relative staining intensity of $\mathrm{S} 100 \mathrm{~B}(1.28 \pm 0.66$ vs. $0.42 \pm 0.31$; $\mathrm{P}=0.01), \mathrm{TNF}-\alpha(2.17 \pm 0.63$ vs. $1.02 \pm 0.61 ; \mathrm{P}=0.02)$ and $\mathrm{IL}-1 \beta$ ( $2.01 \pm 0.50$ vs. $1.11 \pm 0.50 ; \mathrm{P}=0.02)$ expression levels were significantly increased in the patients with OA compared with Control patients. Correlation analysis revealed a significant correlation between S100B and TNF- $\alpha(r=0.42 ; \mathrm{P}=0.04$; Fig. 1B) and between S100B and IL-1 $(\mathrm{r}=0.73 ; \mathrm{P}=0.001$; Fig. 1C).

Increased expression levels of inflammatory cytokines in synovial joint fluid in OA model rabbits. A rabbit model of
Table III. Quantification of mRNA expression levels of S100B, TNF- $\alpha$ and IL- $1 \beta$ in human OA tissues.

\begin{tabular}{lccc}
\hline Gene name & OA $(n=23)$ & Control $(\mathrm{n}=20)$ & P-value \\
\hline S100B & $1.28 \pm 0.66$ & $0.42 \pm 0.31$ & 0.01 \\
TNF- $\alpha$ & $2.17 \pm 0.63$ & $1.02 \pm 0.61$ & 0.02 \\
IL-1 $\beta$ & $2.01 \pm 0.50$ & $1.11 \pm 0.50$ & 0.02 \\
\hline
\end{tabular}

$\mathrm{IL}-1 \beta$, interleukin $1 \beta$; OA, osteoarthritis; TNF- $\alpha$, tumor necrosis factor $\alpha$.

OA was established and the cartilage tissue and synovial fluid were collected for disease assessment. H\&E staining revealed an increased cartilage tissue destruction; there was decreased SO staining of the superficial cartilage layer, as well as increased Mankin score, which indicated successful establishment of OA model (Fig. 2A and B, respectively). ELISA analysis was performed to quantify the level of inflammatory cytokines in synovial fluid, and it was demonstrated that TNF- $\alpha(573.3 \pm 15.4$ vs. $102.6 \pm 8.7$ pg; Fig. $2 C)$ and IL-1 $\beta$ (378.6 \pm 7.2 vs. $170.1 \pm 5.8$ pg; Fig. 2D) expression levels were significantly increased in OA compared with Control rabbits.

S100B expression levels are increased in OA rabbits. Owing to the increased expression levels of inflammatory cytokines in OA rabbits, the possible changes of the genes including S100B, FGF1 and FGFR1 were investigated using OA and Control cartilage tissue. The results demonstrated that S100B mRNA (3.5 \pm 0.8 vs. $1.0 \pm 0.4)$ and protein (1.7-fold) expression levels were significantly increased in OA compared with Control rabbits (Fig. 3A and B, respectively). Significant increases in expression were also observed for FGF1 mRNA $(2.1 \pm 0.7$ vs. $1.0 \pm 0.3)$ and protein (2.1-fold) levels, as well as FGFR1 mRNA $(1.8 \pm 0.7$ vs. $1.0 \pm 0.3)$ and protein (2.0-fold) levels in OA compared with the Control rabbits (Fig. 3A and $\mathrm{B}$ ). In addition, immunohistochemical staining confirmed that the expression levels of S100B were increased and the level of type II collagen was decreased in OA compared with the Control rabbits (Fig. 3C).

S100B regulates inflammatory cytokine secretion in human synovial fibroblasts. Owing to the increased expression levels of S100B and some inflammatory cytokines in OA rabbits, synovial fibroblasts were isolated from normal human cartilage tissues and the expression levels of inflammatory cytokines were analyzed in the condition medium following manipulation of S100B expression by overexpression vector or siRNA transfection (Fig. 4). The results indicated that $\mathrm{S} 100 \mathrm{~B}$ overexpression significantly increased TNF- $\alpha(623.8 \pm 23.8$ vs. $214.5 \pm 14.8$ pg; Fig. $4 B)$ and IL-1 $\beta$ (1101.3 \pm 29.6 vs. $617.5 \pm 37.5$ pg; Fig. 4C) expression levels in LPS-stimulated human synovial fibroblasts compared with Control transfected cells, whereas S100B knockdown decreased TNF- $\alpha(186.2 \pm 16.7$ vs. $214.5 \pm 14.8$ pg; Fig. 4E) and IL-1 $\beta$ (404.1 \pm 15.99 vs. $617.5 \pm 37.5$ pg; Fig. 4F) expression levels in the LPS-stimulated human synovial fibroblasts compared with the Controls. 
A

Control
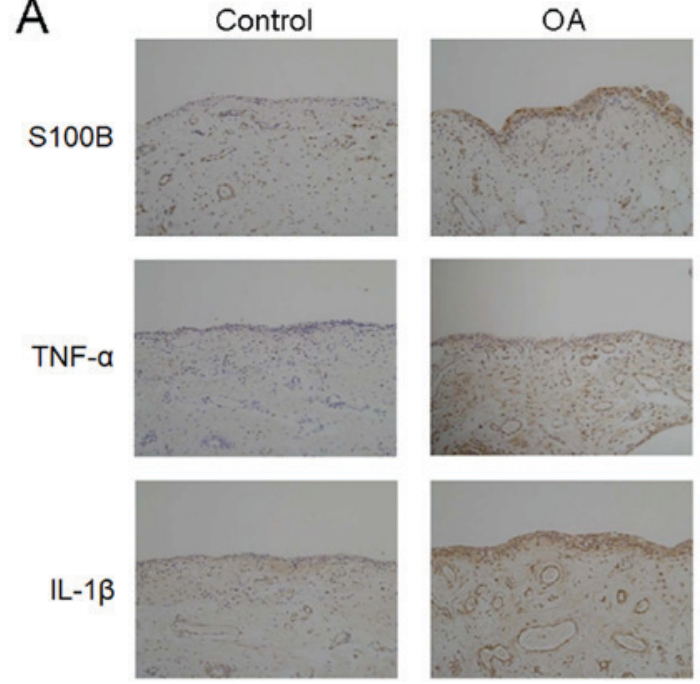

B
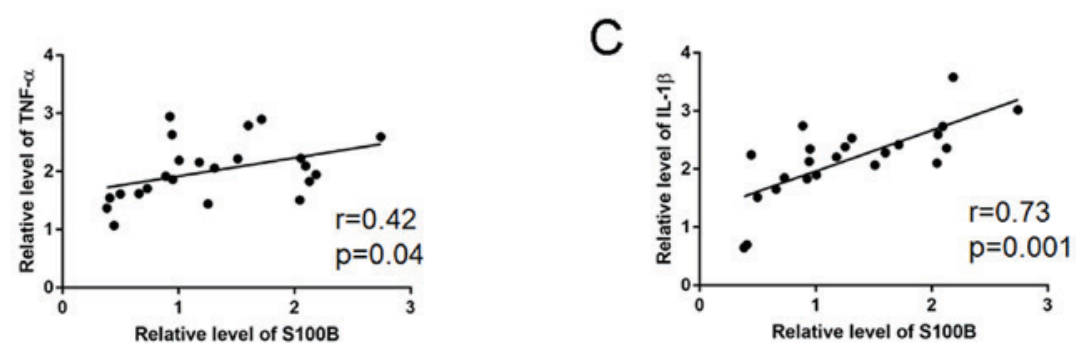

Figure 1. S100B expression levels are increased in patients with OA. (A) Immunohistochemical expression patterns and relative staining intensity quantification of the inflammatory mediators and growth factor in the cartilage tissue of patients with OA and healthy Controls. Correlation analysis between S100B and either (B) TNF- $\alpha$ or (C) IL-1 $\beta$. IL, interleukin; OA, osteoarthritis; TNF, tumor necrosis factor. * P<0.05 vs. Control.

A

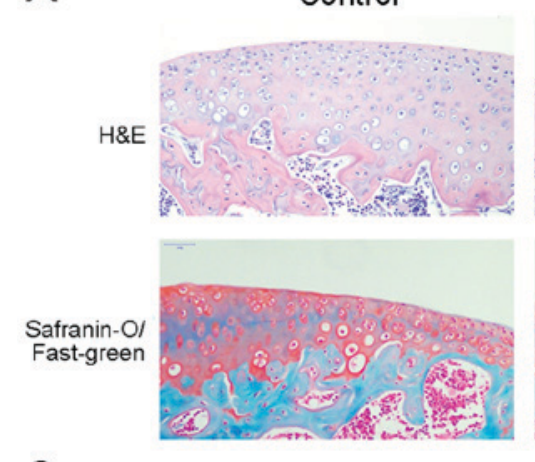

\section{C}

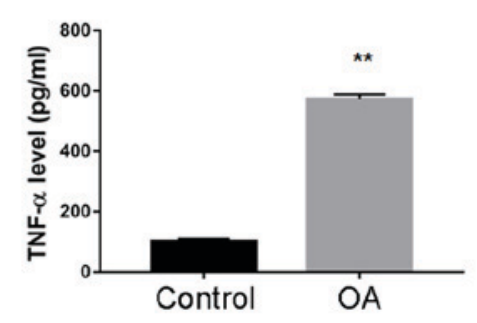

$\mathrm{OA}$
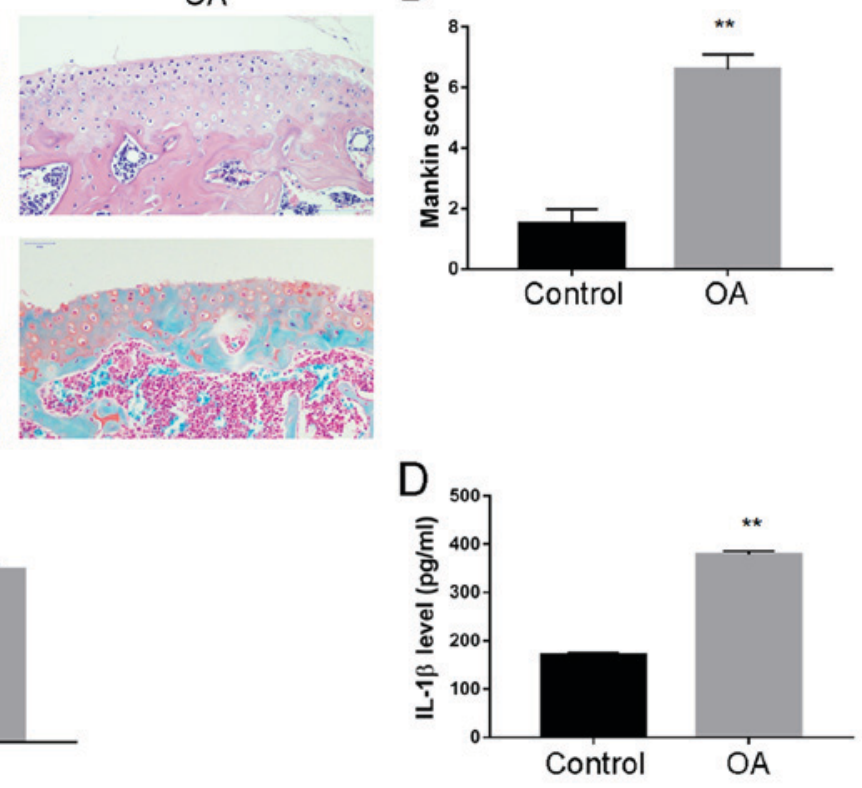

Figure 2. Expression levels of inflammatory cytokines are increased in synovial joint fluid in a OA model rabbits. (A) Histological assessment of OA model by H\&E and Safranin-O/Fast-Green staining. Decreased Safranin-O staining superficial cartilage layer in the OA knees indicated the successful establishment of OA model. (B) Mankin score assessment of OA model. Significantly increased Mankin score was demonstrated in OA compared with the control rabbits. (C) Increased expression levels of TNF- $\alpha$ were demonstrated in OA compared with the control rabbits. (D) Increased expression levels of IL-1 $\beta$ were demonstrated in OA compared with the control rabbits. ${ }^{* *} \mathrm{P}<0.01$ vs. Control. H\&E, hematoxylin and eosin; IL, interleukin; OA, osteoarthritis; TNF, tumor necrosis factor.

FGFR1 is involved in the S100B-mediated inflammation response in LPS-treated synovial fibroblasts. As S100B is an intracellular protein and FGFR1-mediated signaling pathway is involved in the inflammatory effects in synovial 


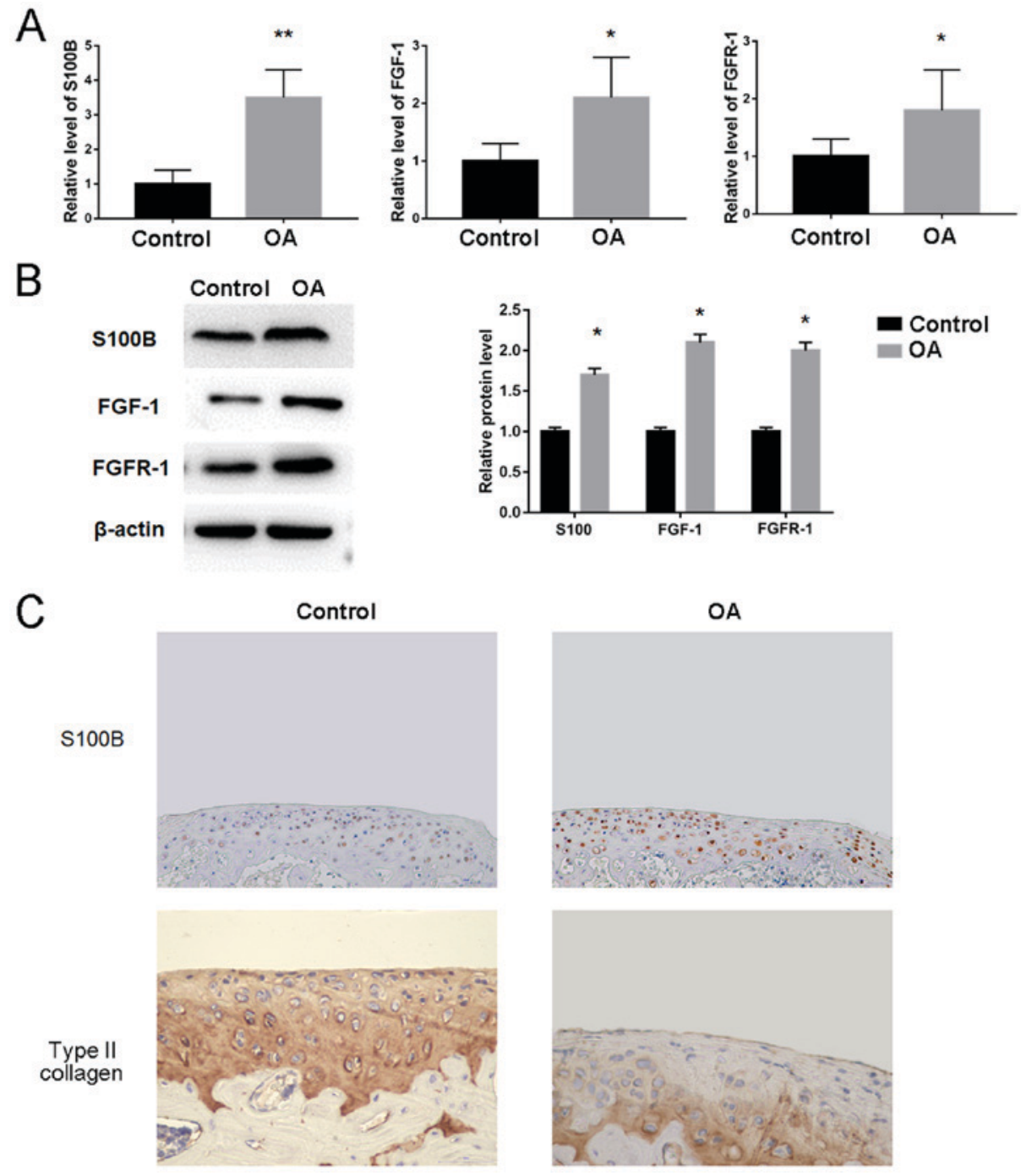

Figure 3. S100B, FGF1 and FGFR1 expression levels are increased in OA model rabbits. (A) Increased expression levels of S100B, FGF1 and FGFR1 mRNA in OA rabbits compared with Control rabbits were determined by reverse transcription-quantitative polymerase chain reaction. (B) Increased protein expression levels of S100B, FGF1 and FGFR1 were determined by western blotting. (C) Immunohistochemical analysis demonstrated increased S100B-specific staining and decreased type II collagen staining in OA rabbits compared with Control rabbits. ${ }^{*} \mathrm{P}<0.05$ vs. Control. FGF, fibroblast growth factor; FGFR, FGF receptor; OA, osteoarthritis.

tissues (20), the roles of S100B and FGFR1 were investigated. FGFR1 mRNA and protein expression levels (Fig. 5A and B, respectively) were increased in human synovial fibroblasts cells overexpressing S100B and were significantly decreased in cells treated with S100B siRNA knockdown, compared with Control cells. Furthermore, FGFR1 knockdown was performed (Fig. 5C), and the results of the RT-qPCR analyses revealed significantly decreased levels of FGF1 mRNA in knockdown cells, which confirmed that transfection was successfully performed. In addition, the change of inflammatory cytokine expression into the condition medium of LPS stimulated human synovial fibroblasts was investigated, and the results revealed that increased FGFR1 expression induced by LPS and S100B overexpression was significantly attenuated following treatment with FGFR1 siRNA (Fig. 5D and E). The results demonstrated that FGFR1 knockdown significantly decreased TNF- $\alpha(197.9 \pm 16.7$ vs. $631 \pm 33$ pg; Fig. 5 F $)$ and IL-1 $\beta$ (588.7 \pm 33.1 vs. $1173 \pm 30$ pg; Fig. $5 \mathrm{G})$ secretion levels in LPS-stimulated S100B-overexpressing human synovial fibroblasts.

\section{Discussion}

In the present study, cartilage tissue and synovial fluid from patients with OA and a rabbit model of OA, as well as human synovial fibroblasts were used to investigate the role of S100B in the inflammatory response during OA. The results demonstrated that increased expression levels of the inflammatory cytokines TNF- $\alpha$ and IL- $1 \beta$ in OA rabbits were accompanied with significantly increased expression of S100B, FGF1 and FGFR1 at the mRNA and protein level. Furthermore, S100B overexpression and knockdown revealed its regulatory role on the production of TNF- $\alpha$ and IL-1 $\beta$ in LPS-stimulated human synovial fibroblasts. In addition, it was also demonstrated that FGFR1-mediated signaling pathway may be involved in the effects of S100B on inflammatory cytokines. To the best of our knowledge, this is the first study to explore the role of S100B in inflammatory response regulation during OA.

The S100 protein family includes 24 members that function as intracellular and/or extracellular regulators (21). Within 
A

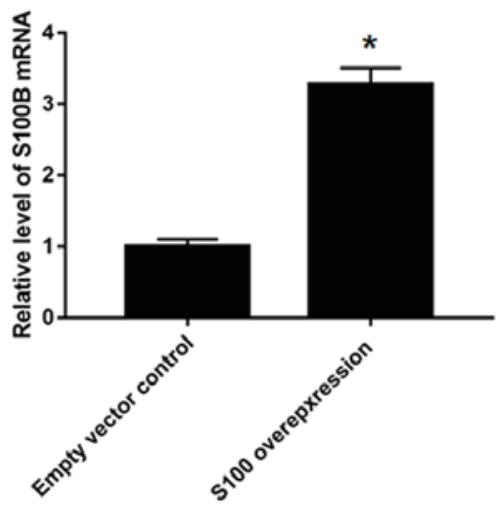

D

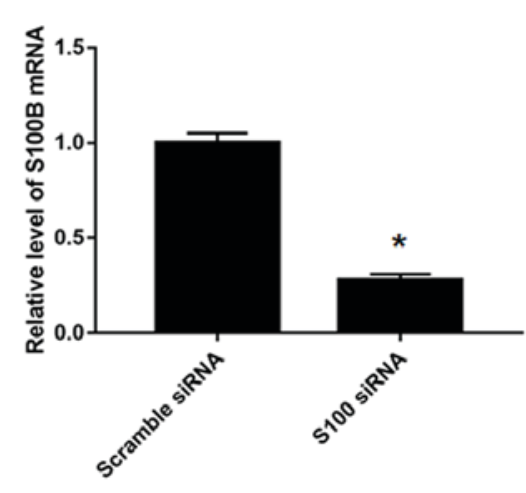

B

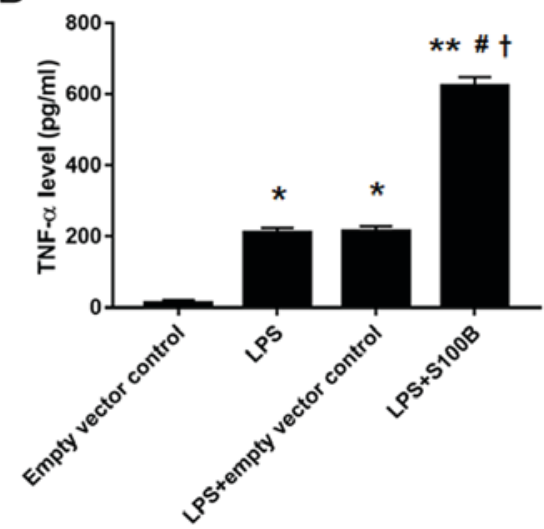

E

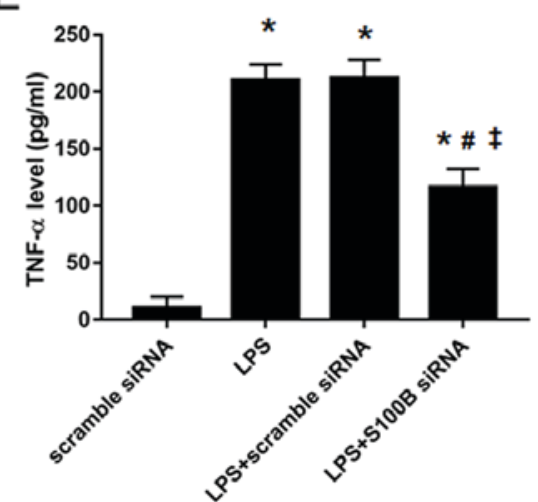

C

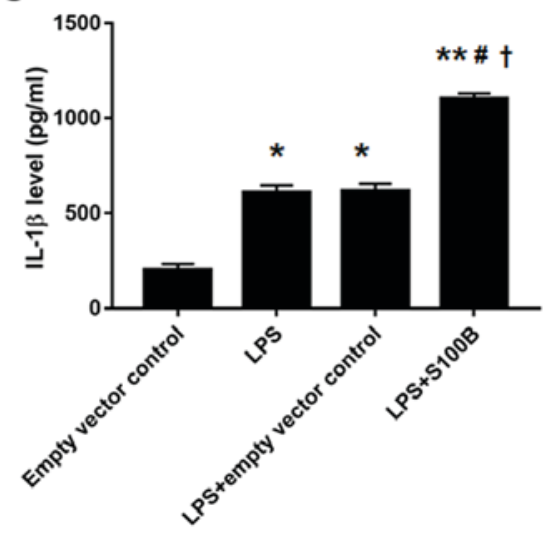

F

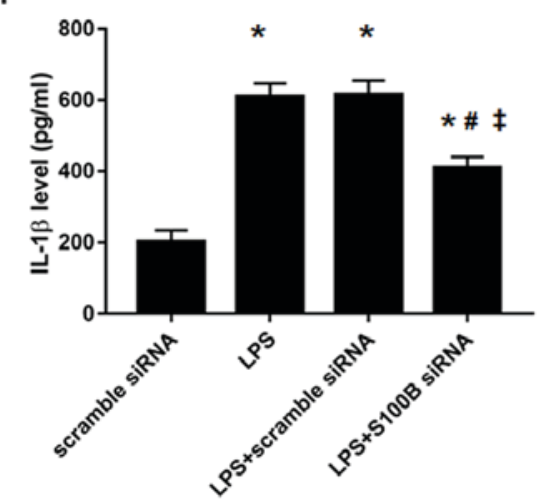

Figure 4. S100B regulates inflammatory cytokine expression levels in human synovial fibroblasts. (A) S100B overexpression was confirmed by RT-qPCR. (B) Increased TNF- $\alpha$ levels in S100B-overexpressing, LPS-stimulated fibroblast, as measured by ELISA. (C) Increased expression levels of IL-1 $\beta$ in S100B-overexpressing LPS-stimulated fibroblasts, as measured by ELISA. (D) S100B knockdown was confirmed by RT-qPCR. (E) TNF- $\alpha$ levels were reduced in LPS-stimulated fibroblasts transfected with S100B siRNA. (F) IL-1 $\beta$ levels were reduced in LPS-stimulated fibroblasts transfected with S100B siRNA. "P<0.05 vs. Empty vector or Scrambled siRNA controls; ${ }^{* *} \mathrm{P}<0.01$ vs. Empty vector; ${ }^{*} \mathrm{P}<0.05$ vs. LPS group; ${ }^{\dagger} \mathrm{P}<0.05$ vs. LPS + Empty vector; ${ }^{\ddagger} \mathrm{P}<0.05$ vs. LPS + scramble siRNA. IL, interleukin; LPS, lipopolysaccharide; RT-qPCR, reverse transcription-quantitative polymerase chain reaction; siRNA, small interfering RNA; TNF- $\alpha$, tumor necrosis factor.

cells, S100 proteins serve important roles in multiple biological processes, including cell proliferation, differentiation, apoptosis, $\mathrm{Ca}^{2+}$ homeostasis, energy metabolism, inflammation and migration/invasion through interactions with a variety of target proteins (such as enzymes, cytoskeletal subunits, receptors, transcription factors and nucleic acids). $\mathrm{S} 100 \mathrm{~B}$ is expressed in chondrocytes (22) and synovial fibroblasts (23). S100B has also been associated with chronic inflammation conditions such as rheumatoid arthritis, diabetes and cystic fibrosis (24); however, its role in inflammation regulation during OA has not yet been explored. In the present study, it was demonstrated that S100B mRNA and protein expression levels were increased in OA rabbits and $\mathrm{S} 100 \mathrm{~B}$ overexpression and knockdown experiments indicated a regulatory role on the production of TNF- $\alpha$ and IL- $1 \beta$ in LPS-stimulated human synovial fibroblasts.

FGFR contain an extracellular ligand-binding domain and an intracellular tyrosine kinase domain, and are single-pass transmembrane receptors that belong to the receptor tyrosine kinases (RTK) family (25). Activation of RTKs by their corresponding ligands (such as FGFs) activate kinases that subsequently initiate intracellular signaling networks that regulate key cellular processes such as cell proliferation, growth, differentiation, migration and survival $(25,26)$.
Furthermore, the spatial and temporal expression of ligands and receptors, as well as the binding specificity between ligands and receptors may affect FGF signaling pathway regulation $(27,28)$. Specificity of FGFR-mediated signaling depends on the cell type and the maturation stage of the cell, and a number of different signaling pathways are involved, including the mitogen-activated protein kinase pathway (extracellular signal-regulated kinases 1 and 2, p38, and c-Jun N-terminal kinases), the phosphoinositide 3-kinase/Akt pathway, and the phospholipase $C \gamma$ pathway (29). As FGF ligands are involved in articular cartilage maintenance, it is reasonable to postulate that FGFRs may also serve a crucial role in articular cartilage homeostasis. Notably, highly expressed FGFR1 and FGFR3 in human articular chondrocytes have been implicated in cartilage metabolism (30). In myoblasts, extracellular S100B was demonstrated to modulate differentiation, stimulate proliferation and reduce apoptosis in vitro by engaging its multi-ligand receptor, receptor for advanced glycosylation end-products, or enhancing basic-FGF (bFGF)-FGFR1 signaling, depending on myoblast density $(31,32)$. In early acute injuries, muscles release $\mathrm{S} 100 \mathrm{~B}$ prior to bFGF and high mobility group box protein (HMGB)-1, and this initial expression diminishes over time, suggesting that S100B may regulate the initial 
A

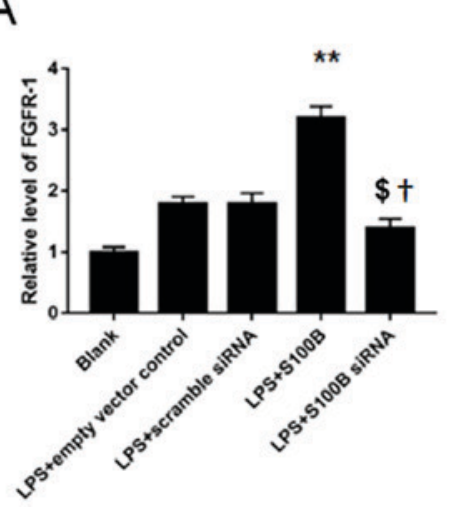

B

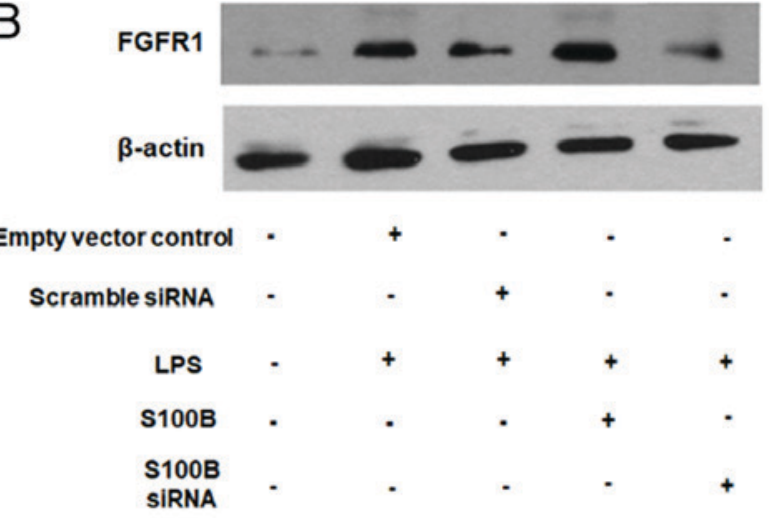

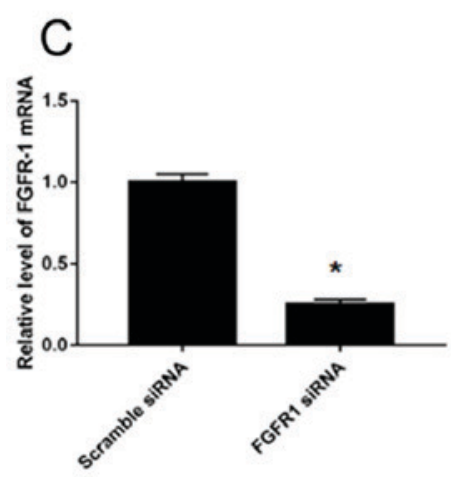

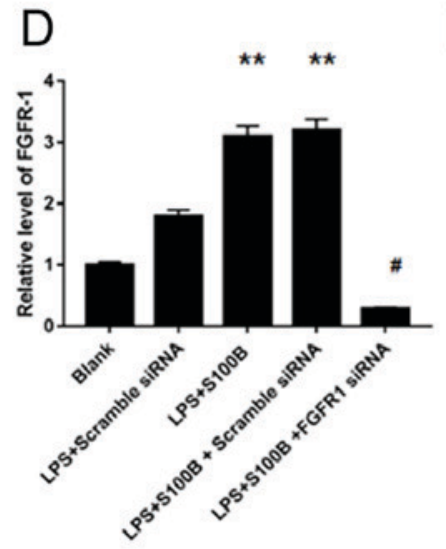

E

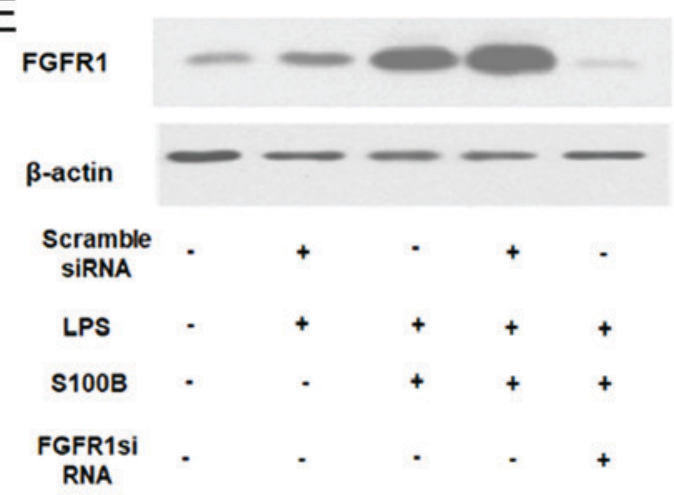

F

G
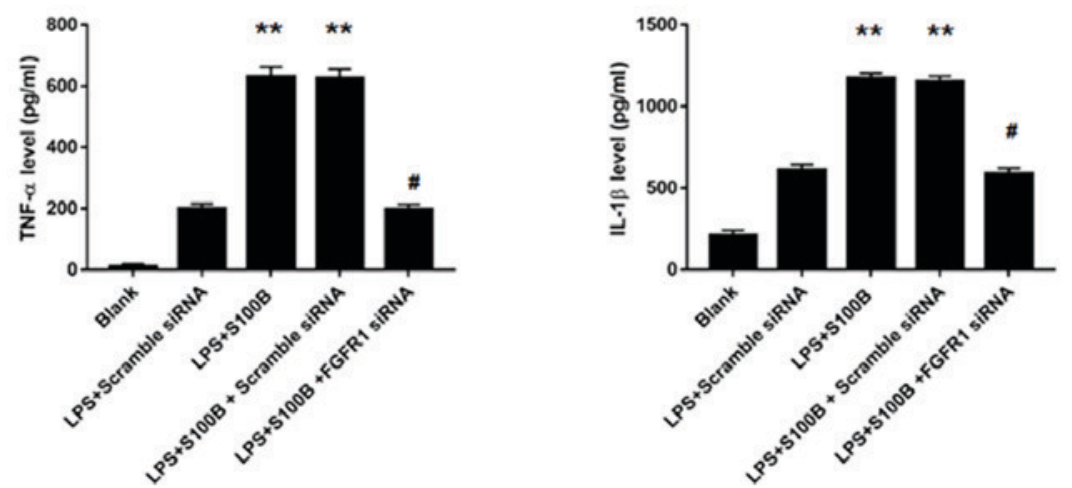

Figure 5. FGFR1 mediates the inflammatory effects of S100B. (A) FGFR1 mRNA expression levels were increased or decreased in S100B-overexpressing and S100B-siRNA synovial fibroblasts, respectively, as measured by RT-qPCR. (B) FGFR1 protein expression levels were increased or decreased in S100B-overexpressing and S100B-siRNA synovial fibroblasts, respectively, as measured by western blotting. (C) Validation of the FGFR1 knockdown FGFR1, by RT-qPCR. (D) RT-qPCR and (E) western blotting were used to determine FGFR1 expression levels in LPS-simulated S100B overexpressed synovial fibroblasts. Increased FGFR1 expression induced by LPS and S100B overexpression was significantly attenuated by FGFR1 siRNA. (F) TNF- $\alpha$ expression levels were decreased following FGFR1 knockdown in LPS-simulated, S100B-overexpressing synovial fibroblasts. (G) IL-1 $\beta$ expression levels were decreased following FGFR1 knockdown in LPS-simulated, S100B-overexpressing synovial fibroblasts. ${ }^{* *} \mathrm{P}<0.01$ vs. LPS + Empty vector or LPS + Scrambled siRNA; ${ }^{\dagger} \mathrm{P}<0.05$ vs. LPS + Empty vector; ${ }^{\$} \mathrm{P}<0.05$ vs. LPS + Scrambled siRNA; ${ }^{\# P}<0.05$ vs. LPS + S100B + Scrambled siRNA. IL-1 $\beta$, interleukin $1 \beta ;$ FGFR, fibroblast growth factor receptor; LPS, lipopolysaccharide; RT-qPCR, reverse transcription-quantitative polymerase chain reaction; siRNA, small interfering RNA; TNF- $\alpha$, tumor necrosis factor.

phases of the regeneration process (24). According to a previous study, synovial macrophages and fibroblasts were demonstrated to actively secrete HMGB1 in collagen-induced arthritis and in hypoxic conditions (33). These similarities between myoblasts and bone tissues may indirectly support the association between S100B and FGFR1 in synovial macrophages. The present results indicated that FGF1 and FGFR1 expression levels were significantly increased during
OA and were consistent with the pattern of increased S100B expression. Furthermore, a previous study demonstrated that silencing of FGFR1 in adult mouse articular chondrocytes inhibits cartilage degeneration progression (34). Knockdown FGFR1 in S100B-overexpressing human synovial fibroblasts in the present study demonstrated that FGFR1 knockdown may attenuate the inflammatory effects exerted by S100B. These data provided a potential link between FGFR1, S100B 
and inflammatory effects. However, owing to the lack of a purified S100B, whether S100B may also interact with bFGF extracellularly, as previously described (35), were not explored in the present study.

Furthermore, spontaneous OA models, which were not induced via gene modification methods such as knockout mice or animals, would provide the best models to study the aging phenotype as they represent primary OA (36). In the present study an OA model rabbit was established, which is also a spontaneous OA model. Compared with the more expensive genetically modified models (fit for specific gene function investigation) and large animal models (suitable for therapy test) (36), the present model could mimic OA disease progression condition with a lower cost.

In conclusion, it was demonstrated that S100B may be involved in the FGFR1-mediated inflammatory response during OA, which may be considered as a potential therapeutic target; however, further studies are needed to confirm the present findings.

\section{Acknowledgements}

Not applicable.

\section{Funding}

The present study was supported by Science and Technology Plan of Suzhou Municipal Government (grant. no. SYS201502, to Lifan Zhu) and Ke Jiao Xing Wei Plan of Wujiang District (grant no. wwk201704, to Pengcheng Shen).

\section{Availability of data and materials}

The datasets used and/or analyzed during the current study are available from the corresponding author on reasonable request.

\section{Authors' contributions}

LZ, ZW, PS and HY analyzed and interpreted the patient data. LZ, ZW, PS, JZhou, JZen, FW, XZ and HY performed the experiments and analyzed the data. LZ, ZW, PS and HY contributed to the writing of the manuscript. All authors read and approved the final manuscript.

\section{Ethics approval and consent to participate}

The present study was approved by the Institutional Review Board of Soochow University; written informed consent was provided by all participants. The animal study protocol was approved by the Institutional Animal Care and Use Committee of Soochow University and was conducted following the international guidelines for animal experimentation.

\section{Patient consent for publication}

Patients provided written informed consent for publication.

\section{Competing interests}

The authors declare that they have no competing interests.

\section{References}

1. Palazzo C, Nguyen C, Lefevre-Colau MM, Rannou F and Poiraudeau S: Risk factors and burden of osteoarthritis. Ann Phys Rehabil Med 59: 134-138, 2016.

2. Prieto-Alhambra D, Judge A, Javaid MK, Cooper C, Diez-Perez A and Arden NK: Incidence and risk factors for clinically diagnosed knee, hip and hand osteoarthritis: Influences of age, gender and osteoarthritis affecting other joints. Ann Rheum Dis 73: 1659-1664, 2014.

3. Lohmander LS and Roos EM: Clinical update: Treating osteoarthritis. Lancet 370: 2082-2084, 2007.

4. Karsdal MA, Michaelis M, Ladel C, Siebuhr AS, Bihlet AR, Andersen JR, Guehring H, Christiansen C, Bay-Jensen AC and Kraus VB: Disease-modifying treatments for osteoarthritis (DMOADs) of the knee and hip: Lessons learned from failures and opportunities for the future. Osteoarthritis Cartilage 24: 2013-2021, 2016.

5. Goldring MB and Otero M: Inflammation in osteoarthritis. Curr Opin Rheumatol 23: 471-478, 2011.

6. Berenbaum F: Osteoarthritis as an inflammatory disease (osteoarthritis is not osteoarthrosis!). Osteoarthritis Cartilage 21: 16-21, 2013.

7. Sellam $J$ and Berenbaum F: The role of synovitis in pathophysiology and clinical symptoms of osteoarthritis. Nat Rev Rheumatol 6: 625-635, 2010.

8. Sorci G, Bianchi R, Riuzzi F, Tubaro C, Arcuri C, Giambanco I and Donato R: S100B protein, a damage-associated molecular pattern protein in the brain and heart, and beyond. Cardiovasc Psychiatry Neurol 2010: 656481, 2010.

9. Sorci G, Riuzzi F, Arcuri C, Tubaro C, Bianchi R, Giambanco I and Donato R: S100B protein in tissue development, repair and regeneration. World J Biol Chem 4: 1-12, 2013.

10. Yammani RR: S100 proteins in cartilage: Role in arthritis. Biochim Biophys Acta 1822: 600-606, 2012.

11. Yammani RR, Carlson CS, Bresnick AR and Loeser RF: Increase in production of matrix metalloproteinase 13 by human articular chondrocytes due to stimulation with S100A4: Role of the receptor for advanced glycation end products. Arthritis Rheum 54: 2901-2911, 2006.

12. Huang ZY, Stabler T, Pei FX and Kraus VB: Both systemic and local lipopolysaccharide (LPS) burden are associated with knee OA severity and inflammation. Osteoarthritis Cartilage 24: 1769-1775, 2016.

13. Ferdowsian HR and Beck N: Ethical and scientific considerations regarding animal testing and research. PLoS One 6: e24059, 2011.

14. Altman R, Asch E, Bloch D, Bole G, Borenstein D, Brandt K, Christy W, Cooke TD, Greenwald R and Hochberg M: Development of criteria for the classification and reporting of osteoarthritis. Classification of osteoarthritis of the knee. Diagnostic and therapeutic criteria committee of the American rheumatism association. Arthritis Rheum 29: 1039-1049, 1986.

15. Pritzker KP, Gay S, Jimenez SA, Ostergaard K, Pelletier JP, Revell PA, Salter D and van den Berg WB: Osteoarthritis cartilage histopathology: Grading and staging. Osteoarthritis Cartilage 14: 13-29, 2006.

16. Ogura N, Tobe M, Sakamaki H, Kujiraoka H, Akiba M, Abiko Y and Nagura H: Interleukin-1beta induces interleukin-6 mRNA expression and protein production in synovial cells from human temporomandibular joint. J Oral Pathol Med 31: 353-360, 2002.

17. Ji Y, Strawn TL, Grunz EA, Stevenson MJ, Lohman AW, Lawrence DA and Fay WP: Multifaceted role of plasminogen activator inhibitor-1 in regulating early remodeling of vein bypass grafts. Arterioscler Thromb Vasc Biol 31: 1781-1787, 2011.

18. Chang J, Tang L, Lei H, Zhang XG, Zuo Z, Huang W and Fu H: Effects of lentiviral infection of mesenchymal stem cells on the expression of octamer transcription factor 4. Mol Med Rep 10: 2249-2254, 2014.

19. Livak KJ and Schmittgen TD: Analysis of relative gene expression data using real-time quantitative PCR and the 2(-Delta Delta $\mathrm{C}(\mathrm{T}))$ method. Methods 25: 402-408, 2001.

20. Gupta AA, Chou RH, Li H, Yang LW and Yu C: Structural insights into the interaction of human S100B and basic fibroblast growth factor (FGF2): Effects on FGFR1 receptor signaling. Biochim Biophys Acta 1834: 2606-2619, 2013.

21. Donato R, Cannon BR, Sorci G, Riuzzi F, Hsu K, Weber DJ and Geczy CL: Functions of S100 proteins. Curr Mol Med 13: 24-57, 2013. 
22. Donato R, Sorci G, Riuzzi F, Arcuri C, Bianchi R, Brozzi F, Tubaro $C$ and Giambanco I: S100B's double life: Intracellular regulator and extracellular signal. Biochim Biophys Acta 1793: 1008-1022, 2009.

23. Bo GP, Zhou LN, He WF, Luo GX, Jia XF, Gan CJ, Chen GX, Fang YF, Larsen PM and Wu J: Analyses of differential proteome of human synovial fibroblasts obtained from arthritis. Clin Rheumatol 28: 191-199, 2009.

24. Hofmann MA, Drury S, Fu C, Qu W, Taguchi A, Lu Y, Avila C, Kambham N, Bierhaus A, Nawroth P, et al: RAGE mediates a novel proinflammatory axis: A central cell surface receptor for S100/calgranulin polypeptides. Cell 97: 889-901, 1999.

25. Schlessinger J: Cell signaling by receptor tyrosine kinases. Cell 103: 211-225, 2000.

26. Lemmon MA and Schlessinger J: Cell signaling by receptor tyrosine kinases. Cell 141: 1117-1134, 2010.

27. Turner $\mathrm{N}$ and Grose R: Fibroblast growth factor signalling: From development to cancer. Nat Rev Cancer 10: 116-129, 2010.

28. Dailey L, Ambrosetti D, Mansukhani A and Basilico C: Mechanisms underlying differential responses to FGF signaling. Cytokine Growth Factor Rev 16: 233-247, 2005.

29. Ornitz DM and Itoh N: The fibroblast growth factor signaling pathway. Wiley Interdiscip Rev Dev Biol 4: 215-266, 2015.

30. Tang J, Su N, Zhou S, Xie Y, Huang J, Wen X, Wang Z, Wang Q, $\mathrm{Xu} \mathrm{W,} \mathrm{Du} \mathrm{X,} \mathrm{et} \mathrm{al:} \mathrm{Fibroblast} \mathrm{growth} \mathrm{factor} \mathrm{receptor} 3$ inhibits osteoarthritis progression in the knee joints of adult mice. Arthritis Rheumatol 68: 2432-2443, 2016.
31. Riuzzi F, Beccafico S, Sagheddu R, Chiappalupi S, Giambanco I, Bereshchenko O, Riccardi C, Sorci G and Donato R: Levels of S100B protein drive the reparative process in acute muscle injury and muscular dystrophy. Sci Rep 7: 12537, 2017.

32. Juban G and Chazaud B: Metabolic regulation of macrophages during tissue repair: Insights from skeletal muscle regeneration. FEBS Lett 591: 3007-3021, 2017

33. Bertheloot D and Latz E: HMGB1, IL-1 $\alpha$, IL-33 and S100 proteins: Dual-function alarmins. Cell Mol Immunol 14: 43-64, 2017.

34. Weng T, Yi L, Huang J, Luo F, Wen X, Du X, Chen Q, Deng C, Chen D and Chen L: Genetic inhibition of fibroblast growth factor receptor 1 in knee cartilage attenuates the degeneration of articular cartilage in adult mice. Arthritis Rheum 64: 3982-3992, 2012.

35. Riuzzi F, Sorci G and Donato R: S100B protein regulates myoblast proliferation and differentiation by activating FGFR 1 in a bFGF-dependent manner. J Cell Sci 124: 2389-2400, 2011.

36. Kuyinu EL, Narayanan G, Nair LS and Laurencin CT: Animal models of osteoarthritis: Classification, update, and measurement of outcomes. J Orthop Surg Res 11: 19, 2016.

(i) (5) $\ominus$ This work is licensed under a Creative Commons Attribution-NonCommercial-NoDerivatives 4.0 International (CC BY-NC-ND 4.0) License. 